\title{
Commentary: Is trust an under-researched component of healthcare organisation?
}

\author{
Judith Green
}

Health Services Research Unit, London School of Hygiene and Tropical Medicine, London WC1E 7HT

WC1E 7HT senior lecturer judith.green@ lshtm.ac.uk
The pattern for provision of primary care has changed radically since the early $1980 \mathrm{~s}^{1}$ The number of small, particularly single-handed, practices has declined rapidly, and most patients now receive a complex, often bewildering, range of primary care services from a large multiprofessional group, rather than seeing their usual general practitioner. This shift has far reaching implications for how professionals work together to provide care and on how that care is experienced by patients. Assumptions that by simply increasing practice size patient care would somehow be improved have been undermined by empirical research on the relations between measures of practice organisation and quality of care. There is no simple relation: larger practices apparently perform better than smaller ones on some indicators of clinical quality of care but worse on factors such as access and continuity. ${ }^{2}$ Explaining how organisational factors relate to healthcare outcomes has become a prized, if unattainable, goal for health services research, and in primary care various mediators have been studied to try to understand the effect of practice organisation on quality of care, including list size, booking intervals for routine consultations, and measures of "team climate," a concept relating to how far a team share a vision of organisational goals and procedures. ${ }^{2-4}$

Nevertheless, limits exist to how far survey evidence can shed light on these relations when so little is known about how primary care organisations really work. Detailed qualitative research on workplace settings suggests that the organisational factors that investigators rush to measure are, in practice, complex processes. One example is consultant presence on intensive care units, posited as a predictor of good clinical outcomes. Recent ethnographic research found that it was not consultant presence in itself that was related to factors likely to improve patient care, but rather the nature of relationships a consultant engendered between nursing and clinical staff. ${ }^{5}$ There is a relative paucity of similar evidence on how professionals work together in the new primary care of large practices, with increasingly bureaucratic organisational structures. Branson and Armstrong's study is, then, an important contribution.

Trust is likely to be a key mediator of relationships both between colleagues and between practitioners and their patients, and understanding how it is experienced, discussed, and built into practice systems will be crucial for understanding the contemporary organisation of primary care. Trust in modern society can be defined in two ways. Firstly, the embodied trust in known others, arising from enduring relationships that are embedded in wider social networks. In general practice, this is perhaps typified by the single-handed general practitioner, whose practice has long been part of a local community. Secondly, trust in impersonal regulatory systems, when contrasting demands are made. Here, the systems trusted most are those least contaminated by vested interests, and there is a preference for them to be regulated fairly, dispassionately, and with reference to objective criteria. Hence, perhaps, the resentments of some of the partners in Branson and Armstrong's study, caught between the embodied trust typical of traditional UK general practice and recently emerging bureaucratic systems (with workload rotas and points) that are still too associated with the practice principals and particular interests to be trusted as fair. Trust of both sorts is likely to be central to understanding how primary care organisations operate and thus how high quality care is delivered.

\section{Competing interests: None declared.}

Branson R, Armstrong D. General practitioners' perceptions of sharing workload in group practices: qualitative study. BMJ 2004;329:381-3.

2 Campbell JL, Ramsay J, Green J. Practice size: impact on consultation length, workload, and patient assessment of care. $\mathrm{Br} J$ Gen Pract 2001;51:644-50.

3 Campbell SM, Hann M, Hacker J, Burns C, Oliver D, Thapar A, et al. Identifying predictors of high quality care in English general practice: an observational study. BMJ 2001;323:784

4 Bower P, Campbell S, Bojke C, Sibbald B. Team structure, team climate and the quality of care in primary care: an observational study. Qual Safety Health Care 2003:12:273-9.

5 Carmel SHM. High technology medicine in practice: the organisation of work in intensive care. PhD thesis. London: University of London, 2003.

doi $10.1136 /$ bmj.38174.496944.7C

\section{Corrections and clarifications}

Screening for sickle cell disease and thalassaemia saving lives

We inadvertently overstated by a factor of 10 the estimated number of people in England with sickle cell disorder in this news article by Zosia Kmietowicz (10 July, p 69). The correct estimation is 12500 .

Prospective cohort study of retinal vessel diameters and risk of hypertension

In this paper by Tien Yin Wong and colleagues, an error relating to the retinal photographs persisted to publication (10 July, pp 79-82). Clearly, for the caption to be correct, the photographs need to be the other way around-that is, the one on the left (not the right) shows narrowed retinal arterioles, and the one on the right shows normal retinal arterioles.

Getting ethics into practice

During the editing process of this editorial by Michael J Parker, we tried to be helpful by adding, in the third paragraph, an explanation of what the Tuskegee study entailed (17 July, p 126). Unfortunately, our explanation was not strictly correct. The Tuskegee study was an observational study of patients who had contracted syphilis; it did not involve the deliberate infection of patients (which is what our explanation suggested). 